\title{
Absolute Calibration of Radar Reflectivity Using Redundancy of the Polarization Observations and Implied Constraints on Drop Shapes
}

\author{
JONATHAN J. GOURLEY \\ NOAA/National Severe Storms Laboratory, Norman, Oklahoma \\ ANTHONY J. ILLINGWORTH \\ University of Reading, Reading, United Kingdom \\ PIERRE TABARY \\ Direction des Systèmes d'Observation, Météo-France, Trappes, France
}

(Manuscript received 11 April 2008, in final form 9 October 2008)

\begin{abstract}
A major limitation of improved radar-based rainfall estimation is accurate calibration of radar reflectivity. In this paper, the authors fully automate a polarimetric method that uses the consistency between radar reflectivity, differential reflectivity, and the path integral of specific differential phase to calibrate reflectivity. Complete instructions are provided such that this study can serve as a guide for agencies that are upgrading their radars with polarimetric capabilities and require accurate calibration. The method is demonstrated using data from Météo-France's operational C-band polarimetric radar. Daily averages of the calibration of radar reflectivity are shown to vary by less than $0.2 \mathrm{~dB}$. In addition to achieving successful calibration, a sensitivity test is also conducted to examine the impacts of using different models relating raindrop oblateness to diameter. It turns out that this study highlights the suitability of the raindrop shape models themselves. Evidence is shown supporting the notion that there is a unique model that relates drop oblateness to diameter in midlatitudes.
\end{abstract}

\section{Introduction}

The accuracy of radar-based rain rates is limited by the calibration of radar reflectivity $Z_{H}$, which must be measured within $1 \mathrm{~dB}$ for rainfall estimates to have an accuracy of $15 \%$. Several approaches to radar calibration have been undertaken and are summarized in Atlas (2002). The receive component of the radar can be calibrated using a transmitter with a known signal strength. Transmit and receive components can be calibrated jointly by positioning a reflective target with a known radar cross section into the radar beam using aircraft, a balloon, etc. Another approach is to compare radar reflectivity to disdrometer measurements, as in Joss et al. (1968). The relative calibrations of the U.S. Weather

Corresponding author address: Jonathan J. Gourley, National Weather Center, University of Oklahoma, 120 David L. Boren Blvd., Norman, OK 73072-7303.

E-mail: jj.gourley@noaa.gov
Surveillance Radar-1988 Doppler (WSR-88D) have also been explored by comparisons with spaceborne radar (Bolen and Chandrasekar 2000) and neighboring WSR-88D radars (Gourley et al. 2003). None of these approaches has emerged as the standard procedure for calibrating radars.

Gorgucci et al. (1992) first noted the self-consistency of $Z_{H}$, differential reflectivity, $Z_{\mathrm{DR}}$, and the range derivative of the differential propagation phase $\Phi_{\mathrm{DP}}$ (or specific differential phase $K_{\mathrm{DP}}$ ) in rain and suggested a calibration method based on adjusting $Z_{H}$ so that rainfall $R$ derived from $Z_{H}$ and $Z_{\mathrm{DR}}$ agreed with $R$ derived from $K_{\text {DP. }}$. Following this pioneering work, Goddard et al. (1994) and Scarchilli et al. (1996) showed that in theory $K_{\mathrm{DP}}$ can be estimated from observations of $Z_{H}$ and $Z_{\mathrm{DR}}$, integrated to yield $\Phi_{\mathrm{DP}}$, and then compared to observed $\Phi_{\mathrm{DP}}$ values; differences are attributed to miscalibration of $Z_{H}$. Methods to calibrate radar reflectivity using the consistency principle have been demonstrated by Gorgucci et al. (1992), Goddard et al. (1994), Illingworth 
and Blackman (2002), Vivekanandan et al. (2003), and Ryzhkov et al. (2005).

Goddard et al. (1994) and Illingworth and Blackman (2002) formulated the consistency relation as the ratio of $K_{\mathrm{DP}}$ to $Z_{H}$ as a function of $Z_{\mathrm{DR}}$. The consistency relation is derived from a normalized gamma drop size distribution (DSD) and makes use of scaling properties between $Z_{H}, K_{\mathrm{DP}}$, and the normalized concentration parameter of the DSD. To formulate a closed relationship between $K_{\mathrm{DP}}, Z_{H}$, and $Z_{\mathrm{DR}}$ from a three-parameter gamma DSD, one must either fix the shape parameter $\mu$ or randomly cycle through a discrete set of variations in parameter ranges (Ulbrich 1983). Illingworth and Blackman (2002) demonstrated that the consistency relation was well behaved and, more importantly, virtually independent of variations in $\mu$. Vivekanandan et al. (2003) simplified the three-parameter DSD representation by relating the shape and slope parameters $(\mu-\Lambda)$ of the gamma distribution from disdrometer observations to yield a closed form of the consistency relation. Although the equations to estimate $K_{\mathrm{DP}}$ from observations of $Z_{H}$ and $Z_{\mathrm{DR}}$ are slightly different from Illingworth and Blackman (2002), their calibration procedure-that is, estimating $K_{\mathrm{DP}}$, integrating it in the radial direction, and comparing to observed $\Phi_{\mathrm{DP}}$ values-is essentially the same.

Ryzhkov et al. (2005) used multiple linear regression to relate $Z_{H}, Z_{\mathrm{DR}}$, and $K_{\mathrm{DP}}$. The coefficients of the regression equation were found empirically using a large sample of DSDs from a disdrometer. The empirical approach differs from the approach that relies on a fixed consistency relationship deduced from a normalized gamma DSD, but it was found to be necessary in Oklahoma where drastically different DSDs were discovered for convective rainfall events versus stratiform events. The use of a fixed consistency relation does not account for variations in DSD but rather assumes that the normalized gamma function adequately describes the DSD in natural rain. Although no physical explanations were provided for the discrepancy in the large and small DSDs in Ryzhkov et al. (2005), it is feasible that they are specific to the intense convective storms unique to the region. This method also differs from earlier studies by using areatime integrals of $K_{\mathrm{DP}}$ instead of radial profiles of $\Phi_{\mathrm{DP}}$. Integrating $K_{\mathrm{DP}}$ to yield the total phase change along the path reduces the noise in the $\Phi_{\mathrm{DP}}$ signal, whereas differentiating $\Phi_{\mathrm{DP}}$ gives an even noisier $K_{\mathrm{DP}}$ estimate. Estimating $K_{\mathrm{DP}}$ requires one to choose an adequate rain path over which $\Phi_{\mathrm{DP}}$ increases linearly. Longer (shorter) path lengths yield less (more) noisy values of $K_{\mathrm{DP}}$. If $\Phi_{\mathrm{DP}}$ increases nonlinearly in the path, then, as Gorgucci et al. (1999) showed, $K_{\mathrm{DP}}$ can be biased either negatively or positively.
The study presented here formalizes the $\Phi_{\mathrm{DP}}$-based calibration method originally proposed by Goddard et al. (1994) so that agencies that are upgrading to dualpolarization capabilities can readily calibrate their radars. Careful data quality procedures were developed and are presented here to fully automate the method; manual selection of candidate rays with a large differential phase is no longer required, as was the case in Illingworth and Blackman (2002) and Vivekanandan et al. (2003). Vivekanandan et al. (2003) also examined the sensitivity of their results to two different models describing raindrop oblateness (represented as a drop aspect ratio) to equal-volume spherical diameter. The differing raindrop shape models yielded an average difference in reflectivity biases of $1.2 \mathrm{~dB}$. Auxiliary information from traditional calibration methods was introduced to infer the correctness of the raindrop shape models. In this study, a sensitivity test that relies on the calibration being independent of rainfall rate and $Z_{\mathrm{DR}}$ was carried out on various models relating raindrop aspect ratio to diameter, which ultimately reveals their suitability without the need for auxiliary information. These results suggest that there is a unique model that relates drop oblateness to diameter in natural rain, at least in midlatitudes.

Section 2 outlines the methodology of our approach using the total phase shift in rain to assess the calibration of $Z_{H}$. Error sources that can either offset or enhance the apparent miscalibration in $Z_{H}$ due to biases in the raw, polarimetric variables and influences from nonraining pixels (e.g., ground clutter, insects, hail, partially melted hydrometeors, ice, etc.) are discussed and correction procedures are presented. The French national weather service, Météo-France, has been operating a C-band polarimetric radar in simultaneous transmission and reception mode since the summer of 2004. Details of the radar's operating characteristics are provided in section 3. Moreover, this section demonstrates application of the $Z_{H}$ calibration method using polarimetric observations from six precipitation episodes.

Currently, there is some doubt in the community regarding the correct model to relate drop oblateness to equal-volume spherical diameter, especially for small drops with diameters of $0.5-1.5 \mathrm{~mm}$ (Thurai et al. 2007). Section 4 examines the sensitivity of calibration results to several raindrop shape models used in the literature. As it turns out, the sensitivity test provides an additional constraint on the various drop shape models that have been proposed. Section 4 also supplies the equations for calibration curves valid at the $\mathrm{X}, \mathrm{C}$, and $\mathrm{S}$ bands. The implications of employing a simple linear slope parameter linking drop oblateness to diameter on rainfall rate estimation and attenuation correction schemes are 
discussed. Conclusions and a summary of results are provided in section 5 .

\section{Description of polarimetric method to calibrate $Z_{H}$}

\section{a. Consistency theory}

This study develops an automatic procedure to compare the theoretical change in $\Phi_{\mathrm{DP}}$, which we call $\Delta \Phi_{\mathrm{DP}}^{\mathrm{th}}$, through a rain path in the radial direction to the observed change, $\Delta \Phi_{\mathrm{DP}}^{\mathrm{obs}}$. To compute $\Phi_{\mathrm{DP}}^{\mathrm{th}}$, values of $K_{\mathrm{DP}}^{\mathrm{th}}$ were estimated first given observations of $Z_{H}, Z_{\mathrm{DR}}$, and their relationship as represented by the curves in Fig. 1 . The consistency curves show the redundancy between $Z_{H}, Z_{\mathrm{DR}}$, and $K_{\mathrm{DP}}$, with raindrop shapes being represented by the Brandes et al. (2002, hereafter BZV) model. Raindrop shapes from the BZV model were found to agree well with $2 \mathrm{D}$ video disdrometer observations of water droplets falling $80 \mathrm{~m}$ from a bridge (Thurai and Bringi 2005); their suitability is a working hypothesis which we will return to in section 4 . The $\mathrm{BZV}$ model is represented as follows:

$$
\begin{aligned}
\frac{b}{a}= & 0.9951+2.51 \times 10^{-2}(D)-3.644 \times 10^{-2}\left(D^{2}\right) \\
& +5.303 \times 10^{-3}\left(D^{3}\right)-2.492 \times 10^{-4}\left(D^{4}\right),
\end{aligned}
$$

where $b / a$ represents the ratio of a drop's semiminor axis length to the semimajor axis length (i.e., the drop aspect ratio), $D$ is the equivolume spherical diameter (in $\mathrm{mm}$ ), and the ratio is set to unity for $D<0.5 \mathrm{~mm}$.

Raindrop spectra were modeled with a normalized gamma distribution (Bringi and Chandrasekar 2001) assuming a shape parameter $(\mu)$ setting of 5 :

$$
N(D)=N_{w} f(\mu)\left(\frac{D}{D_{0}}\right)^{\mu} \exp \left[-(3.67+\mu) \frac{D}{D_{0}}\right],
$$

where $D_{0}$ is the equivolumetric median drop diameter (in $\mathrm{mm}$ ) and $N_{w}$ (in $\mathrm{mm} \mathrm{m}^{-3}$ ) is the normalized concentration, defined as

$$
N_{w}=\frac{(3.67)^{4}}{\pi \rho_{w}}\left(\frac{10^{3} W}{D_{0}^{4}}\right),
$$

where $\rho_{w}$ is $1 \mathrm{~g} \mathrm{~cm}^{-3}$ and $W$ is the rainwater content (in $\mathrm{g} \mathrm{m}^{-3}$ ). [Note that $N_{w}$ has also been referred to as $N_{0}^{*}$, as in Testud et al. (2001).] Varying $\mu$ from 0 to 10 altered the consistency curves in Fig. 1 by less than $5 \%$, demonstrating the insensitivity of the technique to changes in the shape of the drop spectra using BZV drops. Goddard et al. (1994) showed that the consistency curves had a $\mu$ dependence for $Z_{\mathrm{DR}}<1.5 \mathrm{~dB}$, with

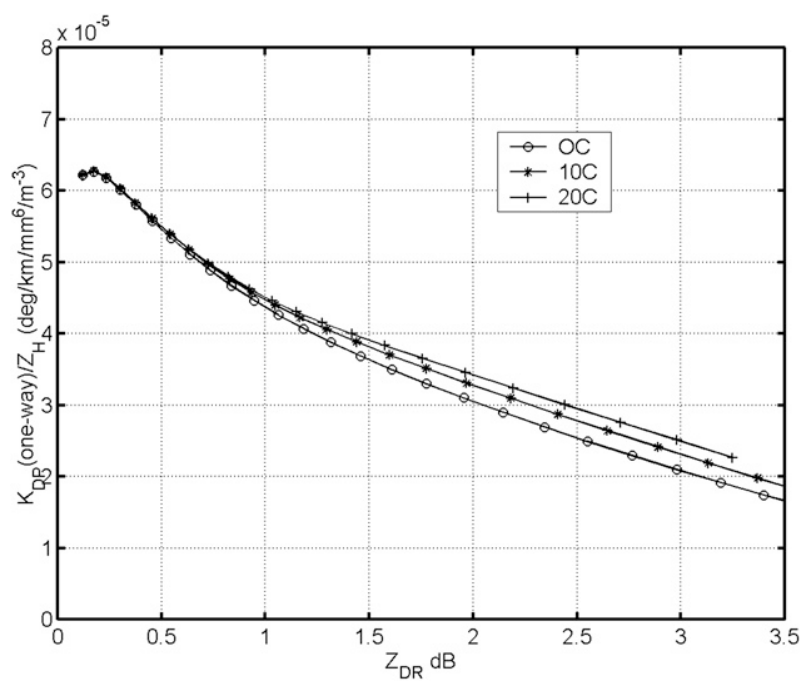

FIG. 1. Consistency curve showing interdependence of $Z_{H}$ $\left(\mathrm{mm}^{6} \mathrm{~m}^{-3}\right), Z_{\mathrm{DR}}(\mathrm{dB})$, and $K_{\mathrm{DP}}$ (one-way, deg $\mathrm{km}^{-1}$ ) at $\mathrm{C}$ band assuming BZV raindrop shapes; their spectra are represented by a normalized gamma distribution with a shape parameter of five. Varying $\mu$ from 0 to 10 altered the consistency curves by less than $5 \%$. Curves are also shown to be insensitive to modeled drop temperature.

differences reaching $25 \%$ for $Z_{\mathrm{DR}}=0.75 \mathrm{~dB}$. However, as we shall see, such variations arise from the physically unrealistic "kink" in the slope of the drop shape model such that drops with $D_{0}<1.1 \mathrm{~mm}$ suddenly become spherical. Consistency curves using drop models without this kink have a much lower $\mu$ dependency. Section 4 addresses the impacts of the drop shape model differences and ultimately their suitability. Note that $N_{w}$ can be interpreted as the intercept value on the concentration axis of an exponential distribution having the same rainwater content as the gamma function. It has the property that $W$ is not a function of the breadth of the distribution $\mu$. Finally, $f(\mu)$ is defined as

$$
f(\mu)=\frac{6}{(3.67)^{4}} \frac{(3.67+\mu)^{\mu+4}}{\Gamma(\mu+4)} .
$$

The polarimetric variables $Z_{H}, Z_{\mathrm{DR}}$, and $K_{D P}$ can be modeled at $\mathrm{C}$ band using the transition $(\mathbf{T})$ matrix formulation of Barber and Yeh (1975). Both $Z_{H}$ and $K_{\mathrm{DP}}$ scale with $N_{w}$, so their ratio is independent of $N_{w}$, as is $Z_{\mathrm{DR}}$. Figure 1 shows that consistency curves of the ratio $K_{\mathrm{DP}} / Z_{H}$ are well-defined functions of $Z_{\mathrm{DR}}$. The different curves correspond to different raindrop temperatures ranging from $0^{\circ}$ to $20^{\circ} \mathrm{C}$. The curves begin to diverge as $Z_{\mathrm{DR}}$ values exceed $1 \mathrm{~dB}$. Theoretical values of $K_{\mathrm{DP}}\left(K_{\mathrm{DP}}^{\mathrm{th}}\right)$ for the $20^{\circ} \mathrm{C}$ range of raindrop temperatures differ by about $10 \%$ at $Z_{\mathrm{DR}}$ values of $2 \mathrm{~dB}$. The sensitivity of consistency relations to raindrop 
TABLE 1. List of error sources in polarimetric radar observations requiring correction prior to application of radar calibration method.

\begin{tabular}{|c|c|}
\hline Problem source & Correction method, threshold, or ray rejection criterion \\
\hline Miscalibration in $Z_{\mathrm{DR}}$ & Calibrate $Z_{\mathrm{DR}}$ using measurements at vertical incidence \\
\hline $\begin{array}{l}\text { Azimuthal dependence of } Z_{\mathrm{DR}} \text { due to } \\
\text { near-radome interference }\end{array}$ & Correct $Z_{\mathrm{DR}}$ using empirical mask \\
\hline Reduced $Z_{H}$ due to water-coated radome & $\begin{array}{l}\text { Reject entire scan if mean } Z_{H} \text { at vertical incidence from } 840 \text { to } 2760 \mathrm{~m} \\
\text { in altitude }>20 \mathrm{dBZ}\end{array}$ \\
\hline Reduced $Z_{H}$ and $Z_{\mathrm{DR}}$ due to attenuation & $\Delta \Phi_{\mathrm{DP}}^{\mathrm{obs}}<12^{\circ}$ \\
\hline Mie scattering effects on $Z_{H}, Z_{\mathrm{DR}}$, and $\Phi_{\mathrm{DP}}^{\mathrm{obs}}$ & Reject ray if a single observation in rain path has $Z_{\mathrm{DR}}>3.5 \mathrm{~dB}$ \\
\hline$\Phi_{\mathrm{DP}}^{\mathrm{obs}}$ at beginning of rain path $\neq 0^{\circ}$ & Find initial $\Phi_{\mathrm{DP}}^{\mathrm{obs}}$ for each ray by computing mean in $6-\mathrm{km}$ window \\
\hline Noisy $\Phi_{\mathrm{DP}}^{\mathrm{obs}}$ in light rain & $\begin{array}{l}\Delta \Phi_{\mathrm{DP}}^{\mathrm{obs}}>10^{\circ} ; \text { rain pathlength }>15 \mathrm{~km} \text {; smooth } \Phi_{\mathrm{DP}}^{\mathrm{obs}} \text { and } \Phi_{\mathrm{DP}}^{\mathrm{th}} \text { in } \\
6 \text {-km window }\end{array}$ \\
\hline Nonprecipitating echoes & $\begin{array}{l}\text { Reject ray if }>5 \% \text { of gates in path were classified as } \\
\text { nonprecipitating pixels using fuzzy logic classification algorithm } \\
\text { (Gourley et al. 2007) }\end{array}$ \\
\hline Presence of hail & Reject ray if a single observation in path has $Z_{H}>50 \mathrm{~dB} Z$ \\
\hline Presence of partially melted or frozen hydrometeors & Range at end of path $<65 \mathrm{~km}$ (or dip in $\rho_{\mathrm{HV}}$ ) \\
\hline
\end{tabular}

temperature and $\mu$ is slight for the BZV drop shapes. A raindrop temperature of $0^{\circ} \mathrm{C}$ was assumed hereafter for the $Z_{H}$ calibration experiment.

Observations of $Z_{H}$ (in $\mathrm{mm}^{6} \mathrm{~m}^{-3}$ ) and $Z_{\mathrm{DR}}$ (in $\mathrm{dB}$ ) and the bottom consistency curve in Fig. 1 were used to provide a value of $K_{\mathrm{DP}}^{\mathrm{th}}\left(\right.$ in $\left.^{\circ} \mathrm{km}^{-1}\right)$ at each range gate. These values were then integrated in the radial direction, thus providing an estimate of $\Phi_{\mathrm{DP}}^{\mathrm{th}}$ at each range gate. The value of $\Phi_{\mathrm{DP}}^{\mathrm{th}}$ at the first range gate is zero by definition; thus, the $\Delta \Phi_{\mathrm{DP}}^{\mathrm{th}}$ from the first range gate to the end of the rain path is the same as $\Phi_{\mathrm{DP}}^{\mathrm{th}}$. Finally, $\Delta \Phi_{\mathrm{DP}}^{\text {th }}$ was compared to $\Delta \Phi_{\mathrm{DP}}^{\mathrm{obs}}$. Differences between the integrated quantities are attributed to miscalibration in $Z_{H}$. This latter inference is subject to the assumptions made to produce the consistency relationship shown in Fig. 1 (i.e., raindrop shape, spectra, and temperature). In addition, biases in observations of $Z_{H}, Z_{\mathrm{DR}}$, and $\Phi_{\mathrm{DP}}$ must be identified and corrected.

\section{b. Correction of biases and spurious signals in polarimetric variables}

Prior to implementing the consistency-based approach to calibrating $Z_{H}$, it is very important to examine the quality of the raw polarimetric quantities. Otherwise, differences between $\Delta \Phi_{\mathrm{DP}}^{\mathrm{th}}$ and $\Delta \Phi_{\mathrm{DP}}^{\mathrm{obs}}$ may be due to effects unrelated to miscalibration in $Z_{H}$. A detailed analysis of polarimetric observations from MétéoFrance's Trappes radar was reported in Gourley et al. (2006). We now list the checks that must be carried out to correct any systematic biases in polarimetric parameters, as well the occasions when rain causes attenuation of $Z_{H}$ and $Z_{\mathrm{DR}}$ and radome attenuation, which must be identified and removed from the analysis. The correction methods are also summarized in Table 1.

Polarimetric measurements at vertical incidence were used for calibrating $Z_{\mathrm{DR}}$. The Trappes antenna points vertically every $15 \mathrm{~min}$ and is rotated $360^{\circ}$ while at zenith. An azimuthal average of $Z_{\mathrm{DR}}$ should be $0 \mathrm{~dB}$ even if the raindrops are canted in the mean or the antenna is wobbling. An analysis performed on measurements collected in the antenna's far field for a 6-h stratiform rainfall event indicated Trappes' $Z_{\mathrm{DR}}$ was biased $0.08 \mathrm{~dB}$ too low. A correction factor of $0.08 \mathrm{~dB}$ has thus been added to all measurements of $Z_{\mathrm{DR}}$. Measurements of $Z_{\mathrm{DR}}$ were also found to be biased as a function of azimuth by as much as $0.4 \mathrm{~dB}$ because of metallic structures within close proximity to the radome. This near-radome interference effect was observed to be repeatable from case to case. An empirical mask was developed and implemented hereafter to offset the biases in $Z_{\mathrm{DR}}$ measurements. After $Z_{\mathrm{DR}}$ was calibrated and corrected due to near-radome interference effects, Gourley et al. (2006) found the expected precision in $Z_{\mathrm{DR}}$ to be $0.2 \mathrm{~dB}$ in rain. The uncertainty in $Z_{\mathrm{DR}}$ calibration is explored further in section 4.

Inspection of a movie loop of $Z_{H}$ when convective echoes passed directly over the radar site revealed a sudden, unrealistic reduction over the entire domain. It is hypothesized that a water-coated radome resulted in the observed power losses. Reductions in $Z_{H}$ and $Z_{\mathrm{DR}}$ due to attenuation were also observed behind intense convective cells. These attenuated measurements were readily recognizable and potentially correctable because of an associated increase in $\Phi_{\mathrm{DP}}^{\mathrm{obs}}$. Power reductions from a wetted radome, however, yielded no increase in $\Phi_{\mathrm{DP}}^{\mathrm{obs}}$. Scans with data that were believed to be influenced by a wetted radome were automatically detected by computing the average $Z_{H}$ at vertical incidence from all azimuths between 840 and $2760 \mathrm{~m}$ in altitude. If the average $Z_{H}$ was greater than $20 \mathrm{~dB} Z$, then the radome was assumed to be wetted. All scans measured within 10 min of the time at which the radome 
was determined to be wetted were discarded from the analysis.

Attenuation and differential attenuation of the signal at $\mathrm{C}$ band are known to reduce measurements of $Z_{H}$ and $Z_{\mathrm{DR}}$ below their intrinsic values. Several correction methods have been proposed and are summarized in Bringi and Chandrasekar (2001). A simple approach linearly relates losses in $Z_{H}$ and $Z_{\mathrm{DR}}$ with increases in $\Phi_{\mathrm{DP}}^{\mathrm{obs}}$, as in Ryzhkov and Zrnic (1995) and Carey et al. (2000). A literature review from the latter study reported mean correction coefficients to be $0.0688 \mathrm{~dB}$ $\left({ }^{\circ}\right)^{-1}$ for $Z_{H}$ and $0.01785 \mathrm{~dB}\left({ }^{\circ}\right)^{-1}$ for $Z_{\mathrm{DR}}$. Significant variability is expected with these coefficients because of changes in raindrop temperature, variability in drop size distribution details, and Mie scattering effects due to large drops or hail (Jameson 1992; Carey et al. 2000; Matrosov et al. 2002, 2005). As opposed to implementing a correction procedure and quantifying its uncertainty, a simple $\Phi_{\mathrm{DP}}^{\mathrm{obs}}$ threshold was implemented to identify and reject rays with data biased by attenuation and differential attenuation effects. Using the literature-mean coefficients reported in Carey et al. (2000) for $\mathrm{C}$ band, a loss of $1 \mathrm{~dB}(0.2 \mathrm{~dB})$ in $Z_{H}\left(Z_{\mathrm{DR}}\right)$ is expected with $\Phi_{\mathrm{DP}}^{\mathrm{obs}}$ $\sim 14.5^{\circ}\left(11.2^{\circ}\right)$. A $\Delta \Phi_{\text {DP }}^{\text {obs }}$ threshold was established at $12^{\circ}$ so that the attenuation in $Z_{H}\left(Z_{\mathrm{DR}}\right)$ ranges from $0(0)$ $\mathrm{dB}$ to a maximum estimate of $1.0(0.2) \mathrm{dB}$ at the end of the path; the average attenuation of the observed $Z_{H}$ $\left(Z_{\mathrm{DR}}\right)$ along the path is reduced to less than $0.5(0.1) \mathrm{dB}$. Regardless, these losses result in bias that will affect the accuracy on calculated $K_{\mathrm{DP}}$ and thus the calibration on $Z_{H}$. Using the data from Fig. 1, we calculated that a 0.1 dB loss in observed $Z_{\mathrm{DR}}$ due to attenuation will yield an estimate of $K_{\mathrm{DP}} / Z_{H}$ (i.e., the ordinate on Fig. 1) that is biased $4 \%-5 \%$ too high. The associated loss in $Z_{H}$, however, causes the ratio to be biased negatively by $11 \%$. The combined result is a $6 \%-7 \%$ negative bias on calculated $K_{\mathrm{DP}}$, resulting in $0.2-0.3 \mathrm{~dB}$ of negative bias in calibrating $Z_{H}$. Accurate attenuation correction with uncertainty estimates could potentially increase the accuracy of the consistency-based $Z_{H}$ calibration method. Data with $\Delta \Phi_{\mathrm{DP}}^{\mathrm{obs}}>12^{\circ}$ were not considered in the analysis.

Mie scattering effects occur with equivolumetric median diameter drops $>2.5 \mathrm{~mm}$ or $Z_{\mathrm{DR}}>2.5-3 \mathrm{~dB}$ at $\mathrm{C}$ band. These large drops can produce differential phase shift on backscatter, leading to transient maxima in $\Phi_{\mathrm{DP}}^{\mathrm{obs}}$, and resonance effects can increase $Z_{D R}$ (Bringi and Chandrasekar 2001). Resonance effects on polarimetric quantities were addressed by rejecting rays if a single gate had $Z_{\mathrm{DR}}>3.5 \mathrm{~dB}$. The combination of the $Z_{\mathrm{DR}}$ threshold with the aforementioned $\Delta \Phi_{\mathrm{DP}}^{\mathrm{obs}}$ threshold adequately eliminated Mie scattering effects on polarimetric variables.

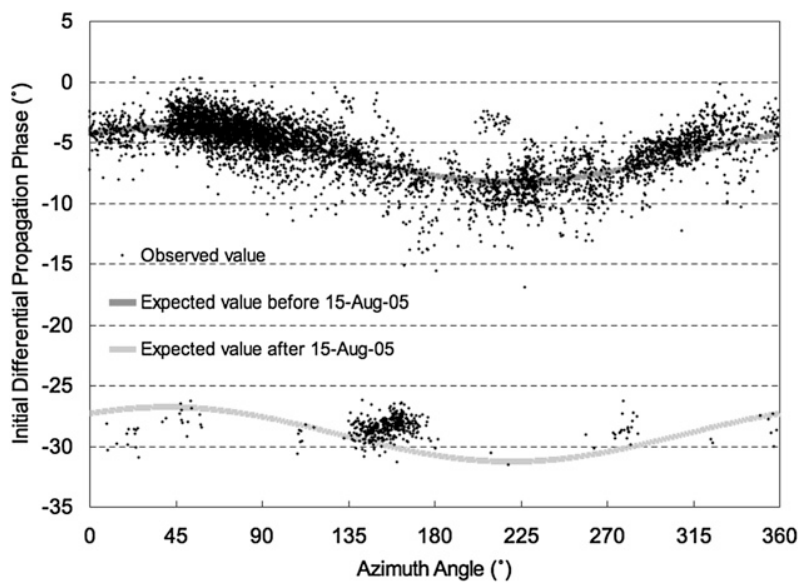

FIG. 2. Scatterplot of initial mean $\Phi_{\mathrm{DP}}^{\mathrm{obs}}$ values plotted as a function of radar azimuth angle. Mean values are computed along the first 25 gates within observations of rain. Observations show a sinusoidal dependence on azimuth angle, which is attributed to the waveguide rotary joint. In addition, the system differential phase changed after the waveguide was replaced on 15 Aug 2005.

Because the goal of the calibration experiment is to compare $\Delta \Phi_{\mathrm{DP}}^{\mathrm{th}}$ and $\Delta \Phi_{\mathrm{DP}}^{\mathrm{obs}}$ at the end of the rain path, it was necessary to retrieve the starting value of $\Phi_{\mathrm{DP}}^{\mathrm{obs}}$ for each ray. Gourley et al. (2006) examined the behavior of initial $\Phi_{\mathrm{DP}}^{\mathrm{obs}}$ values for three different cases. Initial $\Phi_{\mathrm{DP}}^{\mathrm{obs}}$ were biased negatively $6^{\circ}$ and varied with azimuth. The azimuthal dependence was consistent for all three cases and was attributed to the waveguide rotary joint. An empirical mask was developed in Gourley et al. (2006) to correct initial $\Phi_{\mathrm{DP}}^{\mathrm{obs}}$ data so that their starting values were $\sim 0^{\circ}$. In this study, however, greater accuracy in initial $\Phi_{\text {DP }}^{\text {obs }}$ data was needed because the analysis only considered data with $\Delta \Phi_{\mathrm{DP}}^{\mathrm{obs}}<12^{\circ}$. The two sine curves in Fig. 2 show the expected initial $\Phi_{D P}^{\text {obs }}$ values before and after the waveguide was replaced on 15 August 2005. The points cluster around the expected values; however, there is notable scatter of $2^{\circ}-3^{\circ}$. For most applications, such as using $\Phi_{\text {DP }}^{\text {obs }}$ for attenuation correction, an error of $2^{\circ}-3^{\circ}$ is acceptable. In the proposed $Z_{H}$ calibration methodology, a $2^{\circ}-3^{\circ}$ initial $\Phi_{\text {DP }}^{\text {obs }}$ error is $25 \%$. A procedure was therefore developed to retrieve the initial $\Phi_{\mathrm{DP}}^{\mathrm{obs}}$ values for each ray using an arithmetic mean $\Phi_{\mathrm{DP}}^{\mathrm{obs}}$ computed within the first 25 gates $(6 \mathrm{~km})$ of raining pixels, which are shown as points in Fig. 2. The determination of raining versus nonraining pixels is described in section $2 c$. The retrieved $\Phi_{\mathrm{DP}}^{\mathrm{obs}}$ values for each ray are used as the initial values in the rain path rather than the expected values computed from the empirically derived sine curves.

When comparing $\Delta \Phi_{\mathrm{DP}}^{\mathrm{obs}}$ to $\Delta \Phi_{\mathrm{DP}}^{\mathrm{th}}$ at the farthest range gate, where $\Delta \Phi_{\mathrm{DP}}^{\mathrm{obs}}<12^{\circ}$, the inherent noise in $\Phi_{\mathrm{DP}}^{\mathrm{obs}}$ measurements can introduce errors into the comparison. 
Noise also impacts $\Phi_{\mathrm{DP}}^{\text {th }}$ because those values are derived from $Z_{H}$ and $Z_{\mathrm{DR}}$ measurements. Gourley et al. (2006) found the standard deviation of $\Phi_{\mathrm{DP}}^{\mathrm{obs}}$ in rain to be $1.8^{\circ}$ when the copolar cross correlation coefficient at zero time lag, $\rho_{\mathrm{HV}}(0)$, was at least 0.99 . This standard deviation was reduced at most by a factor of 5 if gradients due to nonuniform rainfall path were not present by smoothing $\Phi_{\mathrm{DP}}^{\mathrm{obs}}$ within a 25 -gate window $(6 \mathrm{~km})$ in the radial direction. The same smoothing procedure, a simple running arithmetic mean in 25-gate window, was applied to $\Phi_{\mathrm{DP}}^{\mathrm{th}}$ data. In addition, rain paths were required to be at least $15 \mathrm{~km}$ in length and must have yielded $\Delta \Phi_{\mathrm{DP}}^{\mathrm{obs}}>$ $10^{\circ}$. The use of smoothing and of rain paths greater than $15 \mathrm{~km}$ producing at least $10^{\circ}$ of $\Delta \Phi_{\mathrm{DP}}^{\mathrm{obs}}$ minimizes the impact of noise when comparing single values of $\Delta \Phi_{\mathrm{DP}}^{\mathrm{obs}}$ to $\Delta \Phi_{\mathrm{DP}}^{\mathrm{th}}$ at the end of the rain path.

\section{c. Rejection of rays containing nonrain echoes}

The presence of nonprecipitating targets, predominantly from anomalous propagation and insects, impacted measurements of $Z_{H}, Z_{\mathrm{DR}}, \rho_{\mathrm{HV}}(0)$, and $\Phi_{\mathrm{DP}}^{\mathrm{obs}}$. These common contaminants were found to be associated with relatively low values of $\rho_{\mathrm{HV}}(0)$ as well as with noisy $\Phi_{\mathrm{DP}}^{\text {obs }}$ and $Z_{\mathrm{DR}}$ measurements. A fuzzy logic algorithm described in Gourley et al. (2007) was developed and implemented to discriminate precipitating from nonprecipitating echoes. The developed algorithm employs membership functions that were empirically derived from polarimetric observations of $\rho_{\mathrm{HV}}(0)$, the texture of $\Phi_{\mathrm{DP}}^{\mathrm{obs}}$, and the texture of $Z_{\mathrm{DR}}$. The weight supplied to each polarimetric variable was determined by the areal overlap between the curves representing precipitating and nonprecipitating echoes. The greatest weight was applied to the texture of $\Phi_{\mathrm{DP}}^{\mathrm{obs}}$, meaning this variable is significantly different for precipitating versus nonprecipitating echoes. Each pixel was automatically classified as being either precipitation or nonprecipitation. If more than $5 \%$ of the pixels in a given rain path were determined to be nonprecipitating echoes, then the entire ray was rejected.

Precipitating echoes from the perspective of the fuzzy logic algorithm include pixels containing hail, partially melted hydrometeors, and frozen hydrometeors. Consistency theory, however, is only valid for hydrometeors in liquid phase. Rays that contained a single pixel with $Z_{H}>50 \mathrm{~dB} Z$ were discarded to mitigate the impacts of hail. Measurements within and above the melting layer were avoided by setting a maximum range for the rain path's end point to $65 \mathrm{~km}$. This range was found manually by observing a decrease in $\rho_{\mathrm{HV}}(0)$ with range, an increase and greater fluctuation of $Z_{\mathrm{DR}}$, and an increase in $Z_{H}$. Determining the maximum range at which rain measurements are possible can be easily automated by detecting the bright band, as demonstrated in Gourley and Calvert (2003), Brandes and Ikeda (2004), Giangrande et al. (2005), Tabary et al. (2006), and Matrosov et al. (2007). In this study, the criteria for rejecting rays with potentially frozen hydrometeors were set stringently so that questionable pixels were simply discarded. Each ray meeting the aforementioned criteria was considered a candidate for computing the difference between $\Delta \Phi_{\mathrm{DP}}^{\mathrm{obs}}$ and $\Delta \Phi_{\mathrm{DP}}^{\mathrm{th}}$, with the residual being attributed to miscalibration in $Z_{H}$.

\section{Calibration of $Z_{H}$ for the Trappes $C$-band polarimetric radar}

Data collected during June through September of 2005 by Météo-France's operational radar, situated approximately $30 \mathrm{~km}$ southwest of Paris, are used to evaluate its calibration. The transmitted pulses have a width of $2 \mu \mathrm{s}$, a frequency of $5.64 \mathrm{GHz}$, a peak power of 250 $\mathrm{kW}$, and pulse repetition frequencies of 379,321 , and $305 \mathrm{~Hz}$. The 3-dB beamwidth of the 3.7-m diameter antenna is less than $1.1^{\circ}$. Further details of the radar are provided in Table 2. The radar uses simultaneous transmission and reception of horizontally and vertically polarized waves, so cross-coupling between the orthogonally polarized waves could in theory bias $Z_{\mathrm{DR}}$, but, as pointed out by Ryzhkov and Zrnic (2007), this should be negligible in rain because the net mean canting angle of raindrops is close to zero. Beam blocking was common at the lowest elevation angle of $0.4^{\circ}$, so 484 scans of unblocked data at an elevation angle of $1.5^{\circ}$ on 23,26 , 28, and 30 June, 4 July, and 10 September 2005 were used in the calibration experiment; the resolution of the polar data files was slightly oversampled at $0.5^{\circ}$ in azimuth by $240 \mathrm{~m}$ in range, so a total of 348480 rays were examined. Because most of the rays did not contain rain, 5280 rays met all criteria discussed in section 2 . In practice, we found the calibration method activated for most rays containing rain within $50 \mathrm{~km}$ of the radar.

Figure 3 shows range profiles of $Z_{H}, Z_{\mathrm{DR}}$, raw $\Phi_{\mathrm{DP}}^{\mathrm{obs}}$, and smoothed $\Phi_{\mathrm{DP}}^{\mathrm{obs}}$ for the $228^{\circ}$ azimuth valid at 1015 UTC on 26 June 2005. The first $3 \mathrm{~km}$ of data were deemed to be contaminated by clutter according to the fuzzy logic algorithm described in section 2c; beyond that distance plus 12 gates, the thick gray curve shows $\Phi_{\mathrm{DP}}^{\text {th }}$ as computed from consistency theory using BZV raindrop shapes with a normalized gamma distribution $\left(\mu=5\right.$, drop temperature $\left.=0^{\circ} \mathrm{C}\right)$. At a range of $28.5 \mathrm{~km}$, $\Delta \Phi_{\mathrm{DP}}^{\mathrm{obs}}$ reaches $12^{\circ}$, which is the threshold that was established in section 2 to minimize the effects of attenuation on observations of $Z_{H}$ and $Z_{\mathrm{DR}}$ and thus on $\Phi_{\mathrm{DP}}^{\mathrm{th}}$. The thin gray curves correspond to theoretical phase progressions with $\pm 1 \mathrm{~dB}$ perturbations on $Z_{H}$. At this range, $\Delta \Phi_{\mathrm{DP}}^{\text {th }}$ is $11.4^{\circ}$ whereas the $\Delta \Phi_{\mathrm{DP}}^{\mathrm{th}}$ values with 
TABLE 2. Operating characteristics of the Trappes polarimetric radar* (from Gourley et al. 2006).

\begin{tabular}{|c|c|c|}
\hline & Type & Center-fed paraboloid \\
\hline \multirow[t]{10}{*}{ Antenna } & Diameter & $3.7 \mathrm{~m}$ \\
\hline & Beamwidth $(3 \mathrm{~dB}) \mathrm{H}$ and $\mathrm{V}$ & $<1.1^{\circ}$ \\
\hline & Sidelobe level within $\pm 5^{\circ}(\mathrm{H}$ and $\mathrm{V})$ & $<-25 \mathrm{~dB}$ \\
\hline & Sidelobe levels beyond $10^{\circ}(\mathrm{H}$ and $\mathrm{V})$ & $<-40 \mathrm{~dB}$ \\
\hline & Gain $(\mathrm{H}$ and $\mathrm{V})$ & $>43.8 \mathrm{~dB}$ \\
\hline & Max cross polar isolation & $<-30 \mathrm{~dB}$ \\
\hline & Azimuth travel range & $0^{\circ} \rightarrow 360^{\circ}$ (continuous) \\
\hline & Elevation travel range & $-3^{\circ} \rightarrow 183^{\circ}$ \\
\hline & Azimuth-elevation pointing accuracy & $\pm 0.1^{\circ}$ \\
\hline & Azimuth-elevation velocity & $\mathrm{Up}$ to $36^{\circ} \mathrm{s}^{-1}$ \\
\hline \multirow[t]{5}{*}{ Transmitter } & Peak power & $250 \mathrm{~kW}$ \\
\hline & Pulse width & $2 \mu \mathrm{s}$ \\
\hline & Frequency & $5.640 \mathrm{GHz}$ \\
\hline & Wavelength & $5.31 \mathrm{~cm}$ \\
\hline & PRF & Staggered triple-PRT: 379,321 , and $305 \mathrm{~Hz}$ \\
\hline \multirow[t]{2}{*}{ Receiver } & Minimum detectable signal & $<-112 \mathrm{dBm}$ \\
\hline & Total instantaneous dynamic range $(\mathrm{H}$ and $\mathrm{V})$ & $>95 \mathrm{~dB}$ \\
\hline Radar processor & CASTOR2 & \\
\hline
\end{tabular}

* The parameters listed above have been measured by the radar manufacturer.

$\pm 1 \mathrm{~dB}$ perturbations are $14.3^{\circ}$ and $8.4^{\circ}$, respectively. This ray alone suggests that $Z_{H}$ is calibrated within $1 \mathrm{~dB}$; however, additional comparisons between $\Delta \Phi_{\mathrm{DP}}^{\mathrm{obs}}$ and $\Delta \Phi_{\mathrm{DP}}^{\mathrm{th}}$ are needed to draw conclusions with statistical significance.
Comparisons performed on 5280 rays of data over 6 days in a 4-month period are summarized in Fig. 4. The mean and standard error of the mean of the following equation for calibration $(C$; in \%) are shown for each scan, or plan-position indicator (PPI), and for each day:

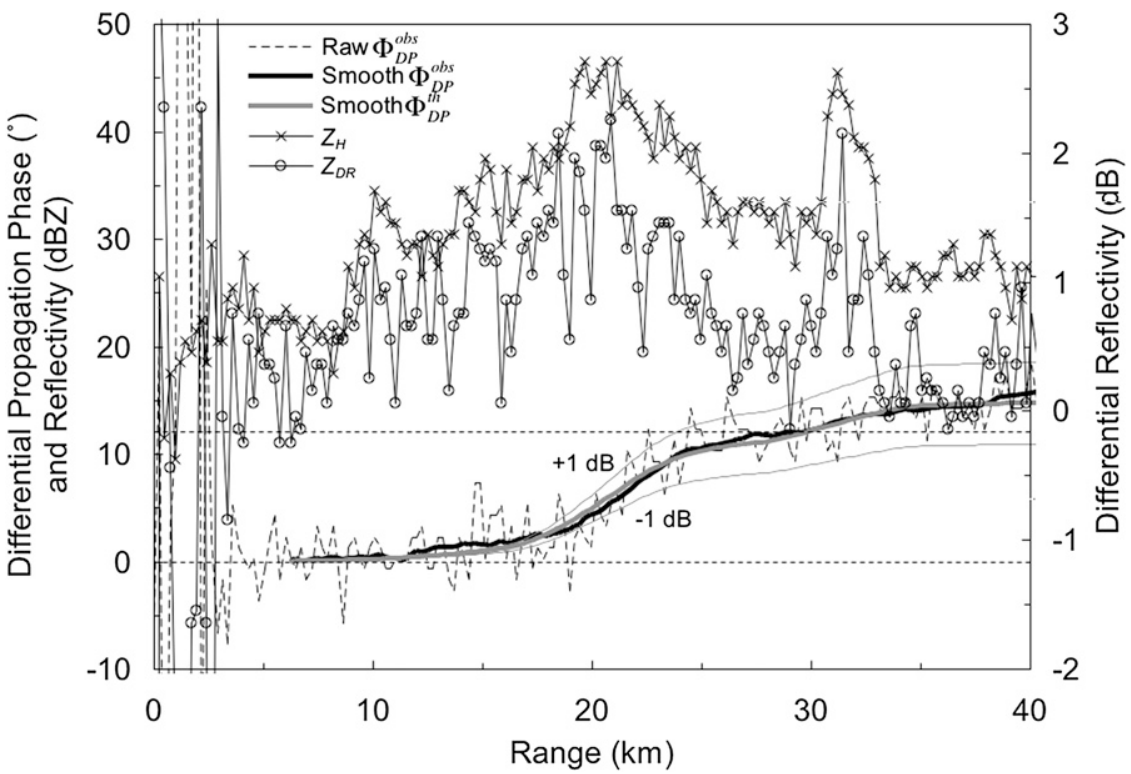

FIG. 3. Radial profiles of observed differential phase shift (dotted gray line), observed differential phase shift smoothed along 25 gates (thick black line), theoretical differential phase shift smoothed along 25 gates (thick gray line), and reflectivity (lines connecting the " $\mathrm{x}$ " symbols) plotted against the left ordinate for the $228^{\circ}$ azimuth valid at 1015 UTC 26 Jun 2005. Theoretical differential phase shifts with +1 - and -1 -dB perturbations in reflectivity are shown as thin gray lines. Differential reflectivity is plotted against the right ordinate and is shown as lines connecting open circles. Horizontal dotted lines correspond to an initial differential phase value of $0^{\circ}$ and final threshold value of $12^{\circ}$. 


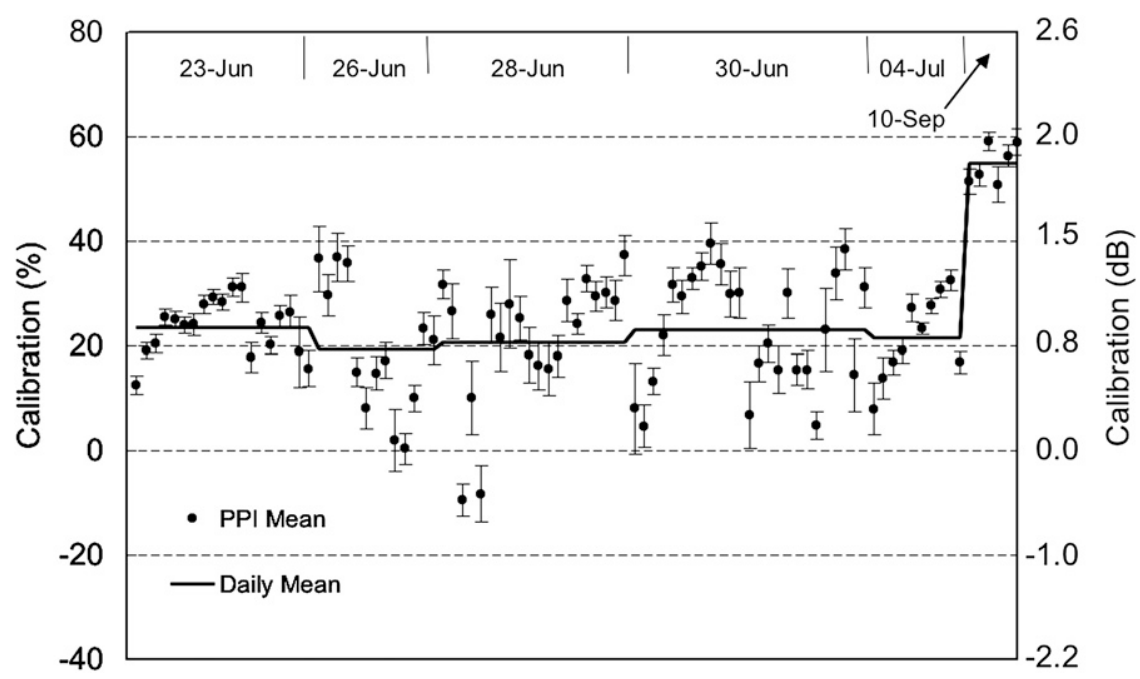

FIG. 4. Calibration of reflectivity ( $\%$ on left ordinate; $\mathrm{dB}$ on right ordinate) for six widespread rain episodes during the summer months of 2005. Mean values are computed for each scan, or plan-position indicator (PPI), and are shown as filled black circles. Error bars correspond to standard error in estimating the mean. Thick black line of daily averaged calibration shows little variability until after the waveguide was replaced on 15 Aug 2005.

$$
C=\frac{\left(\Delta \Phi_{\mathrm{DP}}^{\mathrm{th}}-\Delta \Phi_{\mathrm{DP}}^{\mathrm{obs}}\right) \times 100}{\Delta \Phi_{\mathrm{DP}}^{\mathrm{obs}}} .
$$

The ordinate also indicates the value of $C$ in $\mathrm{dB}$. Although calibration differences from ray to ray were as large as $49 \%(1.7 \mathrm{~dB})$, daily averages varied with time by less than $5 \%(0.2 \mathrm{~dB})$ up to the 10 September 2005 case. Suddenly, the apparent calibration of $Z_{H}$ ( $C$ hereafter) jumped $33 \%(0.9 \mathrm{~dB})$ between 4 July and 10 September 2005. On 18 August 2005, the radar's waveguide was severely damaged and subsequently replaced. This required us to independently recalibrate $Z_{\mathrm{DR}}$, which changed from being biased by $-0.08 \mathrm{~dB}$ before the replacement to $-0.45 \mathrm{~dB}$. The proposed method suggests $Z_{H}$ was biased $22 \%(0.8 \mathrm{~dB})$ too high compared to a calibration based on the radar hardware link budget prior to the waveguide replacement, and the polarimetric calibration technique detected the significant jump in $C$ up to $55 \%(1.7 \mathrm{~dB})$ following the hardware replacement.

\section{Sensitivity of calibration technique to raindrop shape model}

\section{a. Various drop shape models}

Small drizzle drops $(D<0.5 \mathrm{~mm})$ are known to be spherical, whereas the shapes of raindrops become more oblate with increasing diameter. Polarimetric radar measurements serve as the basis for improved rainfall rate estimates, but they rely on the relationship between raindrop aspect ratio and diameter, for which there is no consensus in the polarimetric community. Relatively small errors in the assumed raindrop shape model lead to significant errors in rainfall rate retrievals (Bringi and Chandrasekar 2001, chapter 7).

For many years, raindrop aspect ratios were believed to take on a linear form as a function of drop diameter following the experimental wind tunnel data of Pruppacher and Beard 1970, hereafter PB) for drops larger than 0.5-mm diameter:

$$
\frac{b}{a}=1.03-0.062(D),
$$

where $b / a$ represents the ratio of a drop's semiminor axis length to the semimajor axis length, or drop aspect ratio, and $D$ is the equivolume spherical diameter (in $\mathrm{mm}$ ). Theoretical studies of Green (1975) modeled the balance of forces on a raindrop due to surface tension, hydrostatic pressure, and aerodynamic pressure. Goddard et al. 1982, hereafter GCB) found that the values of $Z_{\mathrm{DR}}$ for drops of diameter $<2.5 \mathrm{~mm}$ measured from a Joss disdrometer exceeded those observed by polarized radar measurements $120 \mathrm{~m}$ above the disdrometer by $0.3 \mathrm{~dB}$, assuming drop aspect ratios follow the linear decrease of (6). They concluded that some modification to the theoretical model of (6) was required and proposed the following empirical raindrop shape model:

$$
\begin{aligned}
\frac{b}{a}= & 1.075-6.5 \times 10^{-2}(D)-3.6 \times 10^{-3}\left(D^{2}\right) \\
& +4.0 \times 10^{-3}\left(D^{3}\right),
\end{aligned}
$$




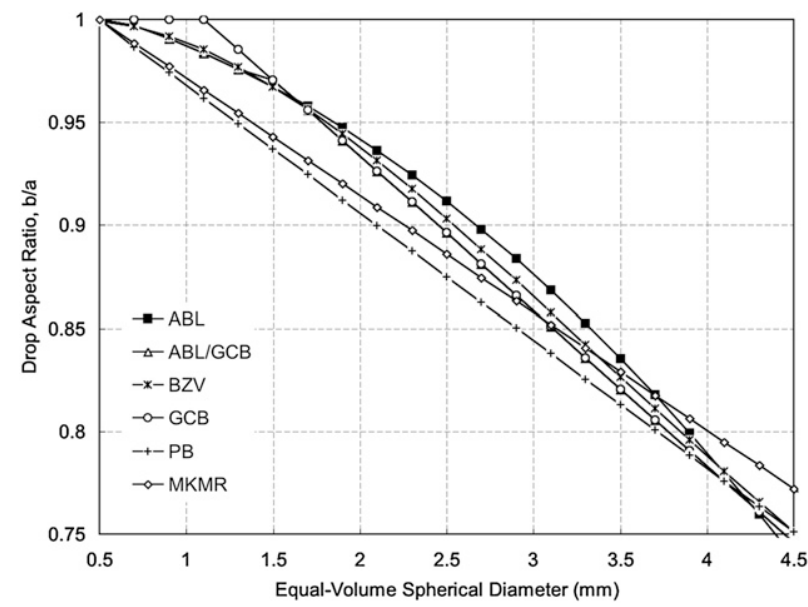

FIG. 5. Various models relating raindrop aspect ratio to equalvolume spherical diameter. Refer to the discussion in section $4 \mathrm{a}$ for details regarding the model descriptions, abbreviations, and formulas.

suggesting that drops with $D<3.5 \mathrm{~mm}$ are much more spherical than predicted by the linear model (Fig. 5).

Results from the simulations of Beard and Chuang (1987) suggested raindrop aspect ratios at equilibrium did not necessarily follow a linear decrease with drop diameter. Chandrasekar et al. (1988) studied natural rainfall using probes onboard aircraft and found drops with diameters 3-4 $\mathrm{mm}$ were in equilibrium. Laboratory studies of Beard and Kubesh (1991) suggested axis ratios of drops with 1.0-1.5-mm diameters were more spherical than equilibrium shapes. The GCB empirical adjustment was essentially confirmed by Andsager et al. (1999, hereafter $\mathrm{ABL}$ ), who conducted careful experiments in long wind tunnels to infer the following drop shapes:

$$
\frac{b}{a}=1.012-1.445 \times 10^{-2}(D)-1.028 \times 10^{-2}\left(D^{2}\right) .
$$

Despite the prevalence of nonlinear drop shape models, Gorgucci et al. (2000) used polarimetric radar to infer raindrop size-shape relationships by treating the 0.062 slope parameter in (6) as a variable, called $\beta$. The so-called $\beta$-retrieval method assumes a variable, linear relationship between drop aspect ratio and diameter. It is assumed that there is no unique drop shape model, and the variability is attributed to asymmetric oscillations excited by collisions and vortex shedding. This method was later incorporated in polarimetric rainfall estimation techniques for S- and X-band radar (Gorgucci et al. 2001; Matrosov et al. 2002) as well as in DSD parameter retrievals (Moisseev et al. 2006). The implications of assuming a linear raindrop shape model are explored in the next section.
BZV proposed the polynomial shown in (1), which is a synthesis of the measurements of Pruppacher and Pitter (1971), Chandrasekar et al. (1988), Beard and Kubesh (1991), and ABL. More recently, Thurai and Bringi (2005) showed excellent agreement of their observations of drop aspect ratios measured by a $2 \mathrm{D}$ video disdrometer of drops falling $80 \mathrm{~m}$ from a railway bridge with the BZV formula in (1). However, the smallest drop size for which they could derive drop aspect ratios was $1.5 \mathrm{~mm}$. There is still some uncertainty of the precise character of drop shapes in range of $0.5-1.5 \mathrm{~mm}$. Section $4 \mathrm{~b}$ evaluates the sensitivity and behavior of calibration results for the proposed raindrop shape models shown in Fig. 5.

Matrosov et al. (2005, hereafter MKMR) estimated values of the $\beta$ variable from polarimetric observations of $Z_{H}, Z_{\mathrm{DR}}$, and $K_{\mathrm{DP}}$ to iteratively correct for attenuation losses in $Z_{H}$ and $Z_{\mathrm{DR}}$. The costliness of the iterative procedure can be avoided by using a constant $\beta$ term, which is believed to have a small impact on final rain rate estimates. The linear model used by MKMR has the following form for drops greater than $0.5-\mathrm{mm}$ diameter (smaller drops are assumed to be spherical):

$$
\frac{b}{a}=(1+0.05 \beta)-\beta D,
$$

with a fixed value of $\beta \approx 0.057 \mathrm{~mm}^{-1}$. This is essentially the same model shown in (6), but for a different slope parameter. In section $4 \mathrm{~b}$, we examine calibration results using the linear drop shape model with two different values for $\beta$ corresponding to (6) and (9).

\section{b. Calibration performance for various drop shapes}

The T-matrix formulation at $\mathrm{C}$ band was used to compute relationships among $Z_{H}, Z_{\mathrm{DR}}$, and $K_{\mathrm{DP}}$ assuming drop spectra are adequately represented by a normalized gamma function with $\mu=5$ and a drop temperature of $0^{\circ} \mathrm{C}$. Figure 6 shows the resulting consistency curves for the proposed raindrop shape models discussed in section 4a and illustrated in Fig. 5. Note that a hybrid model was considered (ABL/GCB), which assumes ABL shapes from $0-1.3 \mathrm{~mm}$ and then GCB for larger drops. The ABL/GCB hybrid model avoids the unrealistic kink in the GCB model at $1.1 \mathrm{~mm}$.

Analysis of drop aspect ratios (Fig. 5) and their resulting consistency curves (Fig. 6) shows that the two linear models of $\mathrm{PB}$ and MKMR yield much higher differential phase shifts (per $Z_{H}$ in $\mathrm{mm}^{6} \mathrm{~m}^{-3}$ ) for an observed $Z_{\mathrm{DR}}$ (in $\mathrm{dB}$ ) because the drops are much more oblate, especially for $D<2.5 \mathrm{~mm}$. The sudden jog in drop shapes to spherical at $D=1.1 \mathrm{~mm}$ in the GCB model results in much less differential phase shift with 


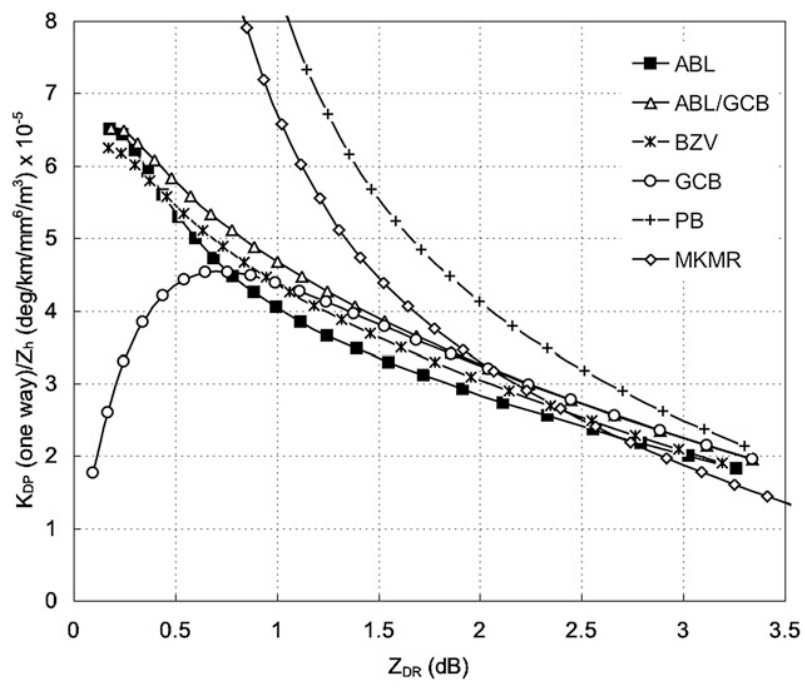

FIG. 6. Consistency curves at $\mathrm{C}$ band for the raindrop shape models illustrated in Fig. 5. Refer to the discussion in section 4a for descriptions of the models and their abbreviations.

small drops for $D<1.55 \mathrm{~mm}$ and is responsible for the fall in the consistency curve for $Z_{\mathrm{DR}}<0.7 \mathrm{~dB}$ in Fig. 6 . Drops described by the ABL model are slightly less oblate than with the other models for $D$ of $1.7-3.7 \mathrm{~mm}$. This less oblate shape results in less differential phase shift for $Z_{\mathrm{DR}}$ observations in the range of $0.8-2.6 \mathrm{~dB}$.

Tests were then carried out to evaluate the sensitivity of apparent radar calibration $[C$; see (5)] to the drop shape models shown in Fig. 5. The polarimetric calibration method described in section 2 was applied to the same dataset described in section 3 , but using the various consistency curves shown in Fig. 6. The calibration of $Z_{H}$ for a radar system should be general such that it is not a function of $D$. Information regarding the suitability of a given drop shape model is revealed upon examination of $C$ as a function of $D$. If $C$ varies with $D$ for a given drop shape model, then there is evidence suggesting the model is inappropriate. It is recognized that $Z_{\mathrm{DR}}$ is related to $\mathrm{D}$ for single drops, and as such could be used to evaluate $C$ as a function of $D$. However, monodispersed raindrop spectra do not occur naturally within a ray, so as a proxy to $D$ we computed $\Phi_{\mathrm{DP}}^{\text {th }}$ only for bins in each ray with $Z_{\mathrm{DR}}>1 \mathrm{~dB}$. Next, we computed $\Phi_{\mathrm{DP}}^{\text {th }}$ for the remainder of the bins in each ray with $Z_{\mathrm{DR}}<1 \mathrm{~dB}$. The sum of the two $\Phi_{\mathrm{DP}}^{\text {th }}$ values is the total differential phase shift estimated from consistency theory whereas the ratio indicates how much theoretical differential phase shift was caused by rayintegrated drops with $Z_{\mathrm{DR}}>1 \mathrm{~dB}$ compared to those with $Z_{\mathrm{DR}}<1 \mathrm{~dB}$. A ratio of 0 indicates all of the $\Phi_{\mathrm{DP}}^{\text {th }}$ was caused by ray-integrated drops with $Z_{\mathrm{DR}}<1 \mathrm{~dB}$ (or small $D)$, a ratio of 1 indicates $\Phi_{\mathrm{DP}}^{\text {th }}$ resulted from an equal proportion of bins with $Z_{\mathrm{DR}}>1 \mathrm{~dB}$ and $Z_{\mathrm{DR}}<1 \mathrm{~dB}$, and a ratio of 5 indicates that a significant contribution of $\Phi_{\mathrm{DP}}^{\text {th }}$ came from rain with $Z_{\mathrm{DR}}>1 \mathrm{~dB}$ (or large $D$ ).

Figure 7 shows histograms of $C$ (in $\mathrm{dB}$ and \%) using the various drop shape models as a function of the ratio of $\Phi_{\mathrm{DP}}^{\text {th }}$ caused by $Z_{\mathrm{DR}}>1 \mathrm{~dB}$ to $\Phi_{\mathrm{DP}}^{\text {th }}$ caused by $Z_{\mathrm{DR}}<$ $1 \mathrm{~dB}$. The bin widths on the abscissa have been chosen to accommodate the relative quantities expressed as a ratio. The mean and standard error of the mean are computed for each ratio bin and are shown as symbols and error bars, respectively. The gray bars plotted against the right ordinate in Fig. 7 show the number of data points contributing to each bin. In addition, thin black lines indicate least squares fit to the unbinned data. The slopes of the lines are computed in terms of $C$ (in \%) per unit ratio of $\Phi_{\mathrm{DP}}^{\text {th }}$ caused by $Z_{\mathrm{DR}}>1 \mathrm{~dB}$ to $\Phi_{\mathrm{DP}}^{\mathrm{th}}$ caused by $Z_{\mathrm{DR}}<1 \mathrm{~dB}$ (dimensionless); thus, the units for the slopes are expressed in $\%$ per dimensionless ratio. The root-mean-square (rms) errors of the linear fits to the curves are also computed in \% per dimensionless ratio. Both the slopes and rms errors are summarized in Table 3.

The relatively large negative slopes of both linear models (PB, $-1.22 \%$; MKMR, $-3.57 \%$ ) indicate a strong dependence of $C$ on the ratio. This result suggests one or a combination of the following: 1) the linear models yield drops that are too oblate for small drops, 2 ) the linear models yield drops that are too spherical for large drops, or 3) observed $Z_{\mathrm{DR}}$ is miscalibrated despite the bias correction steps that were taken in section 2 b. Further analysis of Fig. 5 shows a general convergence of the linear models to the nonlinear ones with increasing ratio, or $D$. Significant differences in drop aspect ratios between linear and nonlinear models are seen at $D<2.5 \mathrm{~mm}$, which indicates that the linear PB and MKMR drop shape models are too oblate for small drops. Lastly, error bars representing the standard error of the mean are larger for the two linear models than for the nonlinear ones, which is indicative of more ray-to-ray variability of $C$.

The ABL model also has a negative slope of $-0.67 \%$ (see Table 3). In this case, Fig. 5 shows that drop aspect ratios with this model are less oblate than the other models for $D$ of 1.7-3.7 mm. Because drop shapes are similar to the other nonlinear models for $D<1.7 \mathrm{~mm}$, we can conclude that the ABL model yields drops that are not oblate enough for medium-sized drops in the range of $1.7-3.7 \mathrm{~mm}$. The GCB model, on the other hand, has a positive slope of $0.81 \%$. Drop aspect ratios from this model are rather more spherical than other nonlinear models for $D<1.5 \mathrm{~mm}$ and have an unrealistic kink at $1.1 \mathrm{~mm}$ (Fig. 5). This oversimplified model yields less $\Phi_{\mathrm{DP}}^{\mathrm{th}}$ for ratios $<1.25$, giving the impression that $C$ is lower for small drops; the positive slope in this case supports the conclusion that the GCB drop shapes 


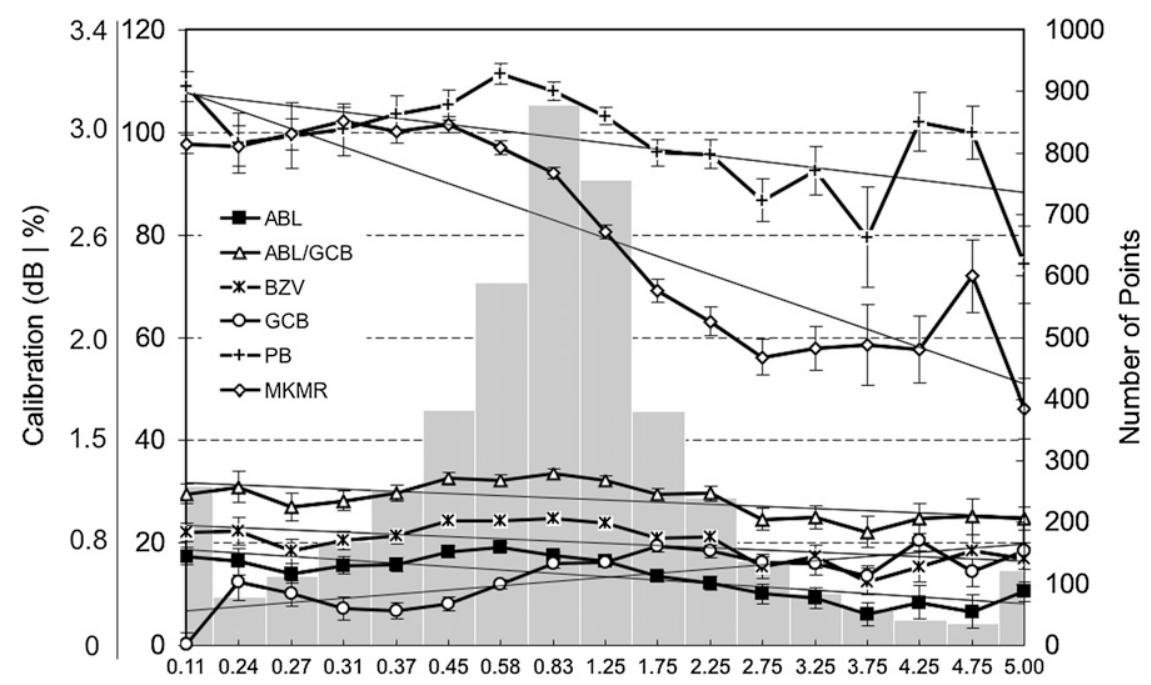

Ratio of $\Phi_{D P}^{\text {th }}$ caused by $Z_{\mathrm{DR}}>1 \mathrm{~dB}$ to $\Phi_{D P}^{\text {th }}$ caused by $\mathrm{Z}_{\mathrm{DR}}<1 \mathrm{~dB}$

FIG. 7. Sensitivity of apparent calibration of $Z_{H}(\mathrm{~dB}$ and $\%$ on left ordinate) as a function of proxy variable to drop diameter for the raindrop shape models illustrated in Fig. 5. Vertical error bars correspond to standard error in estimating the mean at each bin. Thin black lines indicate fits using least squares regression. Sample sizes are shown as gray bars and are plotted against the right ordinate.

are not oblate enough for $D<1.5 \mathrm{~mm}$. Confirmation of this finding is supported by the flatter slope associated with the ABL/GCB model $(-0.43 \%)$. This hybrid model yields drops that are essentially $\mathrm{ABL}$ for small drops $(0-1.3 \mathrm{~mm})$ and GCB thereafter. In essence, the ABL/GCB model "fixes" the oversimplified kink in the GCB model with small drops and produces more oblate drops than the AGL model at intermediate sizes. Calibration results using BZV shapes are also relatively independent of the ratio, with a slope of $-0.44 \%$.

To address the third assertion that miscalibration in $Z_{\mathrm{DR}}$ dictates the slopes, or dependence on $D$, we added positive and negative perturbations of $0.2 \mathrm{~dB}$ to $Z_{\mathrm{DR}}$ observations and then recomputed the curves resulting from each of the drop shape models. This has the effect of nudging the calibration curves in Fig. 6 to the right and left by $0.2 \mathrm{~dB}$. Figure 8 shows curves of the histograms as in Fig. 7 for the drop shape models. The most notable feature in Fig. 8 is the large negative excursions by all drop shape models at low ratios for the $+0.2-\mathrm{dB}$ $Z_{\mathrm{DR}}$ perturbations. The slopes of the calibration curves in Fig. 6 indicate that lower $K_{\mathrm{DP}}^{\text {th }}$ values result from positive $Z_{\mathrm{DR}}$ perturbations, which when integrated in the radial direction give lower $\Delta \Phi_{\mathrm{DP}}^{\mathrm{th}}$ and thus give the impression that the value of $C$ is lower. This effect is more pronounced at low ratios where the slope of the calibration curves is the greatest. In the case of the GCB model, a positive perturbation in $Z_{\mathrm{DR}}$ should yield a higher $\Delta \Phi_{\mathrm{DP}}^{\text {th }}$ and thus higher $C$ at very low ratios cor- responding to $Z_{\mathrm{DR}}<0.7 \mathrm{~dB}$. However, the perturbation itself causes there to be very few data points with $Z_{\mathrm{DR}}<1 \mathrm{~dB}$, so most bins with ratios $<0.5$ are almost unoccupied; accordingly, dashed lines are used in Fig. 8 for these low ratios with sample sizes $<10$ to indicate large errors. Figure 6 indicates that $-0.2-\mathrm{dB} Z_{\mathrm{DR}}$ perturbations should result in higher values of $K_{\mathrm{DP}}^{\mathrm{th}}, \Delta \Phi_{\mathrm{DP}}^{\mathrm{th}}$, and thus $C$. The perturbation has a larger impact at low values of $D$ where the calibration curves are steepest. The blue curves in Fig. 8 show higher values of $C$ from all drop shape models for ratios $<0.58$, which results in steeper slopes of the curves than is shown in Fig. 7. This sensitivity analysis shows that the $Z_{\mathrm{DR}}$ perturbations

TABLE 3. Slopes of linear fits and rms error of fits to curves in Fig. 7 representing calibration of $Z_{H}$ as a function of proxy variable to drop diameter for the different raindrop shape models illustrated in Fig. 5. Refer to the discussion in section 4a for descriptions of the models and their abbreviations. Slopes closest to 0.0 indicate the least sensitivity of apparent radar calibration to drop diameter.

\begin{tabular}{lcc}
\hline \hline Drop shape model & $\begin{array}{c}\text { Slope of least } \\
\text { squares regression (\%) }\end{array}$ & $\begin{array}{c}\text { Rms error of } \\
\text { linear fit }(\%)\end{array}$ \\
\hline ABL & -0.67 & 5.23 \\
ABL/GCB & -0.43 & 3.84 \\
BZV & -0.44 & 4.05 \\
GCB & 0.81 & 6.72 \\
PB & -1.22 & 11.18 \\
MKMR & -3.57 & 26.85 \\
\hline
\end{tabular}




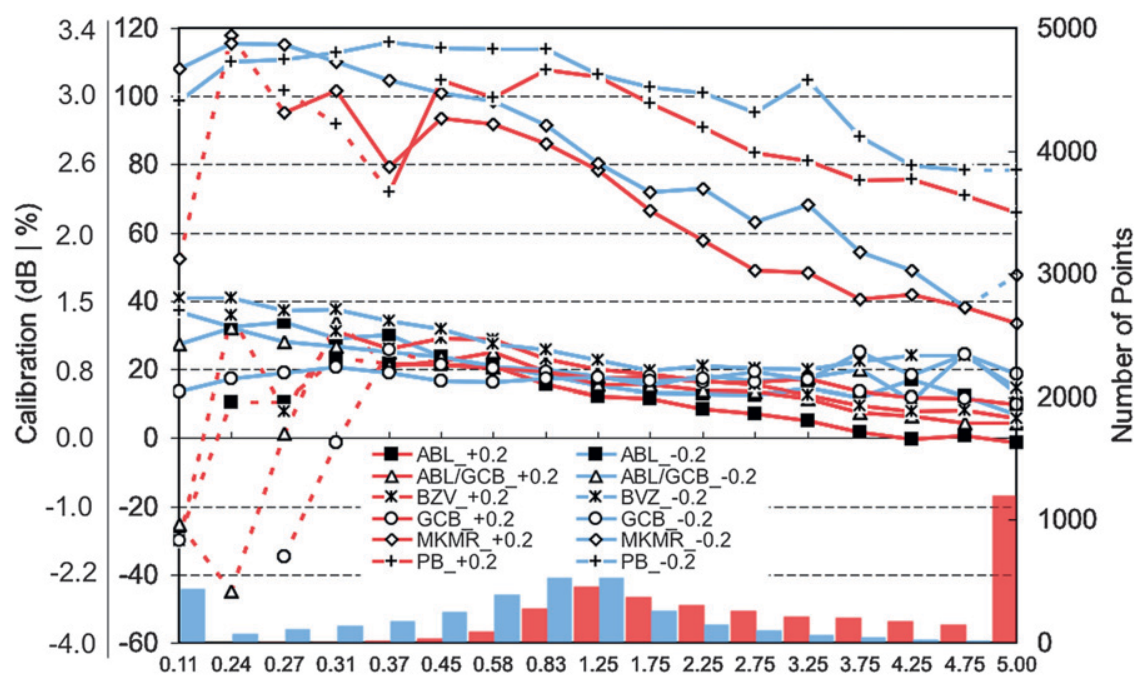

Ratio of $\Phi_{D P}^{\text {th }}$ caused by $Z_{\mathrm{DR}}>1 \mathrm{~dB}$ to $\Phi_{D P}^{\text {th }}$ caused by $\mathrm{Z}_{\mathrm{DR}}<1 \mathrm{~dB}$

FIG. 8. As in Fig. 7, but observed $Z_{\mathrm{DR}}$ values have been perturbed by $\pm 0.2 \mathrm{~dB}$ to illustrate the sensitivity of curves to potential calibration bias in $Z_{\mathrm{DR}}$. Dashed pattern indicates sample sizes were less than 10 . Blue (red) bars correspond to sample sizes of $-0.2(+0.2) \mathrm{dB}$ perturbations and are plotted against the right ordinate.

resulted in changes in the curves that were expected and were more pronounced at low ratios where the calibration curves are steepest. This confirms our assertion that $Z_{\mathrm{DR}}$ was indeed unbiased, and the behavior of the curves shown in Fig. 7 indicates the suitability of the various drop shape models.

The analysis of the dependence of $Z_{H}$ calibration for the various drop shape models on $D$ indicates that the $\mathrm{BZV}$ and $\mathrm{ABL} / \mathrm{GCB}$ models are the most suitable and are virtually indistinguishable. Figure 7 indicates their difference in oblateness for $D$ in the range of 1.7-3.3 mm (Fig. 5) results in $C$ near $8 \%(<0.35 \mathrm{~dB})$. The use of slightly attenuated $Z_{H}$ and $Z_{\mathrm{DR}}$ data adds additional uncertainty of $0.2-0.3 \mathrm{~dB}$. The uncertainty due to drop shape model selection combined with attenuation at $\mathrm{C}$ band yields a $Z_{H}$ calibration accuracy using our proposed method within $0.6 \mathrm{~dB}$.

\section{c. Calibration curves at $X$-, $C$-, and $S$-band frequencies}

The coefficients $a_{i}$ for a third-degree polynomial fit to the BZV calibration curve in Figs. 1 and 6 at $0^{\circ} \mathrm{C}$ of the form

$$
\frac{K_{\mathrm{DP}}}{Z_{H}}=10^{-5}\left(a_{0}+a_{1} Z_{\mathrm{DR}}+a_{2} Z_{\mathrm{DR}}^{2}+a_{3} Z_{\mathrm{DR}}^{3}\right)
$$

are given in Table 4. Here $K_{\mathrm{DP}}$ is one way in deg $\mathrm{km}^{-1}$, $Z_{H}$ is in linear units $\left(\mathrm{mm}^{6} \mathrm{~m}^{-3}\right)$, and $Z_{\mathrm{DR}}$ is in decibels. The calibration curves assume that raindrop shapes are modeled as BZV with a normalized Gamma distribution $\left(\mu=5\right.$; drop temperature $\left.=0^{\circ} \mathrm{C}\right)$. A third-order polynomial in $Z_{\mathrm{DR}}$ provides a fit to within $1 \%$ of the calibration curve shown in Fig. 1. For completeness we also supply the coefficients at $\mathrm{S}$ band $(3.076 \mathrm{GHz})$; the relationship scales slightly more than the frequency for $Z_{\mathrm{DR}}>0.5 \mathrm{~dB}$ because of Mie scattering of the larger drops at $\mathrm{C}$ band $(5.6 \mathrm{GHz})$. The values for $\mathrm{X}$ band $(11.45$ $\mathrm{GHz}$ ) are also given in Table 4; at X band the Mie effects are larger so whereas the calibration values are almost twice those at $\mathrm{C}$ band for $Z_{\mathrm{DR}}<0.5 \mathrm{~dB}$, they are almost the same at $Z_{\mathrm{DR}}=3 \mathrm{~dB}$. For all three frequencies changing $\mu$ from 0 to 10 changes the calibration values by less than $2 \%$, apart from $\mathrm{X}$ band where the exponential curve $(\mu=0)$ is over $2 \%$ higher than the $\mu=5$ curve once $Z_{\mathrm{DR}}>1.5 \mathrm{~dB}$ and reaches $5 \%$ higher for $Z_{\mathrm{DR}}=3 \mathrm{~dB}$.

TABLE 4. Coefficients for a third-degree polynomial fit to the calibration curves for X-, C-, and S-band frequency radars. Refer to (10) in the text for the form of the equation and associated units. The calibration curves assume raindrop shapes are modeled as in $\mathrm{BZV}$ and raindrop spectra follow a normalized gamma distribution $\left(\mu=5\right.$, drop temperature $\left.=0^{\circ} \mathrm{C}\right)$.

\begin{tabular}{lcccr}
\hline \hline Frequency & $a_{0}$ & $a_{1}$ & \multicolumn{1}{c}{$a_{2}$} & \multicolumn{1}{c}{$a_{3}$} \\
\hline $\mathrm{X}$ band & 11.74 & -4.020 & -0.140 & 0.130 \\
$\mathrm{C}$ band & 6.746 & -2.970 & 0.711 & -0.079 \\
$\mathrm{~S}$ band & 3.696 & -1.963 & 0.504 & -0.051 \\
\hline
\end{tabular}




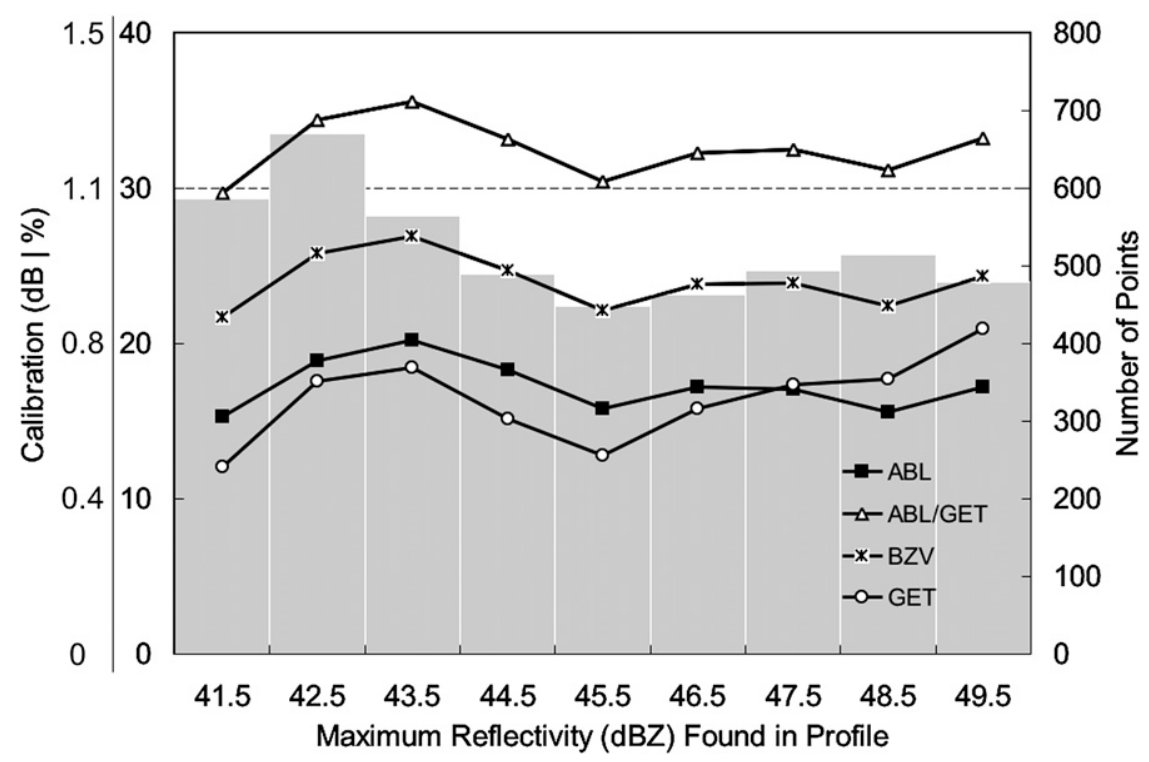

FIG. 9. Sensitivity of apparent calibration of $Z_{H}(\mathrm{~dB}$ and \% on left ordinate) as a function of maximum reflectivity found in each radial for the four valid drop shape models indicated in the legend. Refer to the discussion in section 4a for descriptions of the models and their abbreviations. Sample sizes are shown as gray bars and are plotted against the right ordinate.

\section{d. Implications on methods that retrieve the $\beta$ slope parameter}

Polarimetric radar studies such as Gorgucci et al. (2000), Matrosov et al. (2002), Anagnostou et al. (2004), and Moisseev et al. (2006) have adopted variable raindrop aspect ratio to diameter relationships through the retrieval of the $\beta$ slope parameter. Gorgucci et al. (2006) plot radar observed values of $K_{\mathrm{DP}} / Z_{H}$ against $Z_{\mathrm{DR}}$ as in Fig. 6 and find many data points much closer to the $\mathrm{PB}$ drop shape line than to the curves produced by the ABL, BZV, and GCB drop shape models. It has been hypothesized that raindrop shapes become less oblate because of collisions and vortex shedding, necessitating a variable relationship between drop aspect ratio and drop diameter. These collisions and subsequent asymmetric oscillations should be evident in heavy rainfall where there is increased turbulence. To test this hypothesis, we evaluated $C$ as a function of the maximum $Z_{H}$ found within the rain path. Turbulence should increase with increasing maximum $Z_{H}$, resulting in less oblate drops than predicted from the valid nonlinear models of $\mathrm{ABL}, \mathrm{ABL} / \mathrm{GCB}, \mathrm{BZV}$, and GCB. Note that the models of $\mathrm{PB}$ and MKMR have been eliminated from this analysis because they were shown to be invalid from the analysis in section $4 \mathrm{~b}$. Less oblate drops would have the effect of producing less differential phase shift than predicted from consistency theory and would cause $C$ in (5) to decrease with increasing maximum $Z_{H}$.
Figure 9 shows $C$ plotted as a function of maximum $Z_{H}$ at $1-\mathrm{dB}$ increments in the range of $41-50 \mathrm{dBZ}$. Sample sizes, which are plotted against the right ordinate in gray bars, became too small for maximum $Z_{H}$ bins smaller than $41 \mathrm{~dB} Z$. Rays with maximum $Z_{H}>50$ $\mathrm{dB} Z$ have been eliminated because of potential contamination from hail (see discussion in section $2 \mathrm{c}$ and Table 1). Figure 9 shows little variability of $C$ with maximum $Z_{H}$ in this analysis. We conclude that there is no evidence to suggest that drop shapes fundamentally deviate from the nonlinear models with increasing rain rates where collision frequencies increase.

\section{Discussion and summary}

This study formalizes the method originally proposed by Goddard et al. (1994) to calibrate $Z_{H}$ using the relationship among $Z_{H}, Z_{\mathrm{DR}}$, and the total differential phase shift $\Phi_{\mathrm{DP}}$ along individual radar rays in rain. Development of the method illuminated several data quality issues with the raw variables, which correction procedures were developed to address. The method was then employed to radar observations collected by Météo-France's C-band polarimetric radar located in Trappes. Daily averages of $Z_{H}$ calibration prior to the waveguide replacement were found to be biased $22 \%(0.8 \mathrm{~dB})$ too high and varied by less than $5 \%(0.2 \mathrm{~dB})$. The method detected a sudden jump following the hardware replacement up to $55 \%(1.7 \mathrm{~dB})$. 
A sensitivity test was carried out to determine the impact of different drop shape models on calibration results. The data sample was subdivided into classes based on the amount of theoretical differential phase shift caused by observations with $Z_{\mathrm{DR}}>1 \mathrm{~dB}$ to $Z_{\mathrm{DR}}<1$ $\mathrm{dB}$. This enabled us to examine the stability of $Z_{H}$ calibration as a function of drop size for commonly used drop shape models. This sensitivity test revealed information regarding the suitability of the models themselves, thus providing a constraint on drop shapes. The linear models of PB and MKMR were not supported because they yielded calibration results that depend on drop size. This assertion was confirmed by simulating the impacts of $Z_{\mathrm{DR}}$ bias on the results, which also showed a dependence of the linear drop shape models on drop size. This finding raises concerns for polarimetric attenuation correction and DSD and rainfall retrieval algorithms that rely on a fitted slope parameter relating drop axis ratio to diameter, at least for data collected in midlatitudes. The model proposed by BZV and a hybrid model composed of ABL shapes from $0-1.3 \mathrm{~mm}$ and then GCB thereafter both led to stable calibration results, with much less variability from ray to ray, that were independent of drop size. This consistency over many different rays supports our contention that the natural variability of raindrop spectra is well captured by the use of a normalized gamma function. The difference in calibration of $Z_{H}$ using these two models was $8 \%$ which, when considering the slight attenuation effects on $Z_{H}$ and $Z_{\mathrm{DR}}$, suggests that $Z_{H}$ can be calibrated within $0.6 \mathrm{~dB}$ using the proposed method. The inferred calibration for these drop shapes was unchanged over the range of $Z_{H}$ from 40 to $50 \mathrm{dBZ}$, leading us to question recent suggestions that drop shapes become more spherical in heavy rainfall because of increased turbulence and/or collisions. Results presented in this study support the notion that there is a unique model that relates drop oblateness to diameter for rain in midlatitudes, and the linear drop shape models are not suitable.

The $Z_{H}$ calibration method developed here differs from that proposed by Ryzhkov et al. (2005) in its use of the differential phase shift $\Phi_{\mathrm{DP}}$ along individual, nonattenuated rays instead of $K_{\mathrm{DP}}$ and a fixed, normalized gamma distribution representing drop spectra instead of large statistics of DSDs collected with a disdrometer. The normalized gamma distribution was found to be representative of raindrop spectra observed near Paris and is thus applicable to regions including but not limited to Europe, Japan, and Canada. The proposed calibration method is readily applicable at $\mathrm{X}$ and $\mathrm{S}$ band provided considerations are made for attenuation effects. Polarimetric data collected at S band are less susceptible to attenuation effects; thus, rain paths with larger values of $\Delta \Phi_{\mathrm{DP}}^{\mathrm{obs}}$ of up to $25^{\circ}$ can be used rather than the $12^{\circ}$ threshold that was established for application at $\mathrm{C}$ band. Application at $\mathrm{X}$ band poses more challenges because of similar noisiness in $\Phi_{\mathrm{DP}}^{\mathrm{obs}}$, and a $\Delta \Phi_{\mathrm{DP}}^{\mathrm{obs}}$ of approximately $5^{\circ}$ results in $1 \mathrm{~dB}$ of attenuation in $Z_{H}$; reducing the threshold would limit the calibration accuracy to about $25 \%$ or $1 \mathrm{~dB}$. This problem can be overcome by applying reliable corrections for attenuation to the data such as the combined $\Phi_{\mathrm{DP}}-Z_{\mathrm{DR}}$ constraint that has been adapted for use at X band (Iwanami et al. 2003; Anagnostou et al. 2004; Park et al. 2005). At all wavelengths, the maximum differential phase shift threshold can be increased following improvements to attenuation correction schemes, resulting in the use of longer rain paths.

We believe the proposed $Z_{H}$ calibration method and associated consistency relationships will be useful to agencies that are upgrading their radars with polarimetric capabilities.

Acknowledgments. This work was done in the frame of the PANTHERE Project (Programme ARAMIS Nouvelles Technologie en Hydrometeorologie Extension et Renouvellement) supported by Météo-France, the Ministere de L'Ecologie et du Developpement Durable, the European Regional Development Fund (ERDF) of the European Union, and CEMAGREF.

\section{REFERENCES}

Anagnostou, E. N., M. N. Anagnostou, W. F. Krajewski, A. Kruger, and B. J. Miriovsky, 2004: High-resolution rainfall estimation from X-band polarimetric radar measurements. J. Hydrometeor., 5, 110-128.

Andsager, K., K. V. Beard, and N. F. Laird, 1999: Laboratory measurements of axis ratios for large raindrops. J. Atmos. Sci., 56, 2673-2683.

Atlas, D., 2002: Radar calibration: Some simple approaches. Bull. Amer. Meteor. Soc., 83, 1313-1316.

Barber, P., and C. Yeh, 1975: Scattering of electromagnetic waves by arbitrarily shaped dielectric bodies. Appl. Opt., 14, 2864-2872.

Beard, K. V., and C. Chuang, 1987: A new model for the equilibrium shape of raindrops. J. Atmos. Sci., 44, 1509-1524.

_ , and R. J. Kubesh, 1991: Laboratory measurements of small raindrop distortion. Part II: Oscillation frequencies and modes. J. Atmos. Sci., 48, 2245-2264.

Bolen, S. M., and V. Chandrasekar, 2000: Quantitative cross validation of space-based and ground-based radar observations. J. Appl. Meteor., 39, 2071-2079.

Brandes, E. A., and K. Ikeda, 2004: Freezing-level estimation with polarimetric radar. J. Appl. Meteor., 43, 1541-1553.

—, G. Zhang, and J. Vivekanandan, 2002: Experiments in rainfall estimation with a polarimetric radar in a subtropical environment. J. Appl. Meteor., 41, 674-685.

Bringi, V. N., and V. Chandrasekar, 2001: Polarimetric Doppler Weather Radar: Principles and Applications. Cambridge University Press, $636 \mathrm{pp}$.

Carey, L. D., S. A. Rutledge, D. A. Ahijevych, and T. D. Keenan, 2000: Correcting propagation effects in C-band polarimetric 
radar observations of tropical convection using differential propagation phase. J. Appl. Meteor., 39, 1405-1433.

Chandrasekar, V., W. A. Cooper, and V. N. Bringi, 1988: Axis ratios and oscillations of raindrops. J. Atmos. Sci., 45, 13231333.

Giangrande, S. E., A. V. Ryzhkov, and J. Krause, 2005: Automatic detection of the melting layer with a polarimetric prototype of the WSR-88D radar. Preprints, 32nd Conf. on Radar Meteorology, Albuquerque, NM, Amer. Meteor. Soc., 11R.2. [Available online at http://ams.confex.com/ams/pdfpapers/95894.pdf.]

Goddard, J. W. F., S. M. Cherry, and V. N. Bringi, 1982: Comparison of dual-polarization radar measurements of rain with ground-based disdrometer measurements. J. Appl. Meteor., 21, 252-256.

_, J. Tan, and M. Thurai, 1994: Technique for calibration of meteorological radar using differential phase. Electron. Lett., 30, 166-167.

Gorgucci, E., G. Scarchilli, and V. Chandrasekar, 1992: Calibration of radars using polarimetric techniques. IEEE Trans. Geosci. Remote Sens., 30, 853-858.

,$- \ldots$, and —_, 1999: Specific differential phase estimation in the presence of nonuniform rainfall medium along the path. J. Atmos. Oceanic Technol., 16, 1690-1697.

,,--- , and V. N. Bringi, 2000: Measurement of mean raindrop shape from polarimetric radar observaions. $J$. Atmos. Sci., 57, 3406-3413.

,,--- , and,- 2001: Rainfall estimation from polarimetric radar measurements: Composite algorithms immune to variability in raindrop size-shape relation. J. Atmos. Oceanic Technol., 18, 1773-1786.

—, L. Baldini, and V. Chandrasekar, 2006: What is the shape of a raindrop? An answer from radar measurements J. Atmos. Sci., 63, 3033-3044.

Gourley, J. J., and C. M. Calvert, 2003: Automated detection of the bright band using WSR-88D data. Wea. Forecasting, 18, 585-599.

—_, B. Kaney, and R. Maddox, 2003: Evaluating the calibrations of radars: A software approach. Preprints, 31st Int. Conf. on Radar Meteorology, Seattle, WA, Amer. Meteor. Soc., P3C.1. [Available online at http://ams.confex.com/ams/pdfpapers/ 64171.pdf.]

— , P. Tabary, and J. Parent du Châtelet, 2006: Data quality of the Météo-France C-band polarimetric radar. J. Atmos. Oceanic Technol., 23, 1340-1356.

,-- , and -2007 : A fuzzy logic algorithm for the separation of precipitating from nonprecipitating echoes using polarimetric radar observations. J. Atmos. Oceanic Technol., 24, 1439-1451.

Green, A. W., 1975: An approximation for shapes of large drops. $J$. Appl. Meteor., 14, 1578-1583.

Illingworth, A., and T. Blackman, 2002: The need to represent raindrop size spectra as normalized gamma distributions for the interpretation of polarization radar observations. J. Appl. Meteor., 41, 286-297.

Iwanami, K., E. Le Bouar, J. Testud, M. Maki, R. Misumi, S.-G. Park, and M. Suto, 2003: Application of the rain profiling algorithm "ZPHI" to the X-band polarimetric radar data observed in Japan. Preprints, 31st Int. Conf. on Radar Meteorology, Seattle, WA, Amer. Meteor. Soc., P2B.9. [Available online at http://ams.confex.com/ams/32BC31R5C/techprogram/ paper_64520.htm.]

Jameson, A. R., 1992: The effect of temperature on attenuation-correction schemes in rain using polarization propa- gation differential phase shift. J. Appl. Meteor., 31, 11061118.

Joss, J., J. C. Thams, and A. Waldvogel, 1968: The accuracy of daily rainfall measurements by radar. Preprints, 13th Radar Meteorology Conf., Montreal, QC, Canada, Amer. Meteor. Soc., 448-451.

Matrosov, S. Y., K. A. Clark, B. E. Martner, and A. Tokay, 2002: $\mathrm{X}$-band polarimetric radar measurements of rainfall. J. Appl. Meteor., 41, 941-952.

— D. E. Kingsmill, B. E. Martner, and F. M. Ralph, 2005: The utility of X-band polarimetric radar for quantitative estimates of rainfall parameters. J. Hydrometeor., 6, 248-262.

— K. A. Clark, and D. E. Kingsmill, 2007: A polarimetric radar approach to identify rain, melting-layer, and snow regions for applying corrections to vertical profiles of reflectivity. J. Appl. Meteor. Climatol., 46, 154-166.

Moisseev, D. N., V. Chandrasekar, C. M. H. Unal, and H. W. J. Russchenberg, 2006: Dual-polarization spectral analysis for retrieval of effective raindrop shapes. J. Atmos. Oceanic Technol., 23, 1682-1695.

Park, S.-G., V. N. Bringi, V. Chandrasekar, M. Maki, and K. Iwanami, 2005: Correction of radar reflectivity and differential reflectivity for rain attenuation at $\mathrm{X}$ band. Part I: Theoretical and empirical basis. J. Atmos. Oceanic Technol., 22, 1621-1632.

Pruppacher, H. R., and K. V. Beard, 1970: A wind tunnel investigation of the internal circulation and shape of water drops falling at terminal velocity in air. Quart. J. Roy. Meteor. Soc., 96, 247-256.

— , and R. L. Pitter, 1971: A semi-empirical determination of the shape of cloud and rain drops. J. Atmos. Sci., 28, 86-94.

Ryzhkov, A. V., and D. S. Zrnic, 1995: Precipitation and attenuation measurements at a $10-\mathrm{cm}$ wavelength. J. Appl. Meteor., 34, 2121-2134.

— and - 2007: Depolarization in ice crystals and its effect on radar polarimetric measurements. J. Atmos. Oceanic Technol., 24, 1256-1267.

_ S. E. Giangrande, V. M. Melnikov, and T. J. Schuur, 2005: Calibration issues of dual-polarization radar measurements. J. Atmos. Oceanic Technol., 22, 1138-1155.

Scarchilli, G., E. Gorgucci, V. Chandrasekar, and A. Dobaie, 1996: Self consistency of polarization diversity measurement of rainfall. IEEE Trans. Geosci. Remote Sens., 34, 22-26.

Tabary, P., A. Le Henaff, G. Vulpiani, J. Parent du Châtelet, and J. J. Gourley, 2006: Melting layer characterization and identification with a C-band dual-polarization radar: A long-term analysis. Proc. Fourth European Radar Conf., Barcelona, Spain, ERAD, 17-20.

Testud, J., S. Oury, R. A. Black, P. Amayenc, and X. Dou, 2001: The concept of "normalized" distribution to describe raindrop spectra: A tool for cloud physics and cloud remote sensing. J. Appl. Meteor., 40,1118-1140.

Thurai, M., and V. N. Bringi, 2005: Drop axis ratios from a 2D video disdrometer. J. Atmos. Oceanic Technol., 22, 966-978.

_ , G. J. Huang, V. N. Bringi, W. L. Randeu, and M. Schonhuber, 2007: Drop shapes, model comparisons, and calculations of polarimetric radar parameters in rain. J. Atmos. Oceanic Technol., 24, 1019-1032.

Ulbrich, C. W., 1983: Natural variations in the analytical form of the raindrop size distribution. J. Climate Appl. Meteor., 22, 1764-1775.

Vivekanandan, J., G. Zhang, S. Ellis, D. Rajopadhyaya, and S. Avery, 2003: Radar reflectivity calibration using different propagation phase measurement. Radio Sci., 38, 8049, doi:10.1029/ 2002RS002676. 